\title{
A CONSTRUCTIVE METHOD TO DETERMINE THE VARIETY OF FILIFORM LIE ALGEBRAS
}

\author{
F. J. Echarte, M. C. Márquez, and J. NúÑez, Sevilla
}

(Received October 28, 2004)

\begin{abstract}
In this paper we use cohomology of Lie algebras to study the variety of laws associated with filiform Lie algebras of a given dimension. As the main result, we describe a constructive way to find a small set of polynomials which define this variety. It allows to improve previous results related with the cardinal of this set. We have also computed explicitly these polynomials in the case of dimensions 11 and 12 .
\end{abstract}

Keywords: cohomology of nilpotent Lie algebras, graded filiform Lie algebras, variety of laws of filiform Lie algebras, irreducible component, algorithm

MSC 2000: 17B30

\section{INTRODUCTION}

Filiform Lie algebras were introduced by M. Vergne in the late 60's of the past century [1]. However, before that, Blackburn studied the analogous class of finite $p$-groups and used the term maximal class to call them, which is also now used for Lie algebras [2]. In fact, both the terms filiform and maximal class are synonymous.

Vergne showed that, within the variety of nilpotent Lie multiplications on a fixed vector space, non-filiforms can be relegated to small-dimensional components; thus, from an intuitive point of view, it is possible to consider that quite a lot of nilpotent Lie algebras are filiform, in spite of this last subset not being dense in the space of nilpotent Lie algebras. Apart from that, complex filiform Lie algebras are the most structured subset of nilpotent Lie algebras, with respect to an adapted basis. In this sense, we can study and classify them easier than the set of nilpotent Lie algebras. 
So, in earlier papers, some of us have deeply studied these algebras and obtained quite a lot of results about them. Indeed, we already got the classification of those having dimensions 10, 11 and 12 (see [3], for instance). Moreover, we think that this study can be also considered a small step forward in the problem of the classification of these algebras, although it is not the principal objective of this work.

It is already known that the usual cohomology space of degree 2 of the model filiform Lie algebra with values in its adjoint module is closely related with the study of filiform Lie algebras. For each dimension, it is possible to associate this space with an affine algebraic variety. Polynomials defining this variety are preserved when the dimension increases, although the number of them certainly increases. In fact, all polynomials defining these varieties are homogeneous of degree two and when increasing the degree by one, the only polynomials which are added are linear in the new variables. This suggests that each component of the variety of filiform Lie algebras of dimension $n+1$ may be a bundle over the corresponding component of the variety of dimension $n$ with affine fibres (see [4], [5]).

So, apart from describing the variety, another aim of this paper is to significantly reduce the number of these polynomials, for each dimension. In the paper we also describe an algorithm which allows to determine in a computational way the variety of these algebras. It is suitable for being used in the case of bigger dimensions.

Indeed, this algorithm can be useful for studying the irreducible component of filiform Lie algebras of dimension greater than or equal to 12 , which is actually an open problem. Moreover, some techniques related with Gröbner basis allow us to conjecture that the number of polynomials given by the algorithm could be minimal.

The algorithm can be easily implemented in any symbolic computational package. Concretely, we have used MAPLE in our study.

\section{Cohomology of Lie algebras}

Let $\mathcal{G}$ be a Lie algebra over an arbitrary field $K$ and let $V$ be a $\mathcal{G}$-module. We will denote the $p$-co-chain space of $\mathcal{G}$ having values in $V$ by $C^{p}(\mathcal{G}, V)$, with $p \geqslant 0$. It is the space of $p$-linear alternating mappings of $\mathcal{G}^{p}$ into $V$ for $p \geqslant 1$ and the space of the constant functions from $\mathcal{G}$ into $V$ for $p=0$.

We now consider the coboundary operator $d$, defined as usual starting from the representation of $\mathcal{G}$ associated with $V$ according to

$$
d: C^{p}(\mathcal{G}, V) \longrightarrow C^{p+1}(\mathcal{G}, V)
$$

as

$$
d \varphi(x)=x \varphi \text { for } \varphi \in C^{0}(\mathcal{G}, V), x \in \mathcal{G} \text { if } p=0
$$


and

$$
\begin{aligned}
d \varphi\left(x_{1}, \ldots, x_{p+1}\right)= & \sum_{s=1}^{p+1}(-1)^{s+1}\left(x_{s} \varphi\right)\left(x_{1}, \ldots, \hat{x}_{s}, \ldots, x_{p+1}\right) \\
& +\sum_{1 \leqslant s<t \leqslant p+1}(-1)^{s+t} \varphi\left(\left[x_{s}, x_{t}\right], x_{1}, \ldots, \hat{x}_{s}, \ldots, \hat{x}_{t}, \ldots, x_{p+1}\right)
\end{aligned}
$$

if $p \geqslant 1$ for $\varphi \in C^{p}(\mathcal{G}, V)$ and $x_{1}, \ldots, x_{p+1} \in \mathcal{G}$ where the symbol means that the letter is omitted.

We will denote by $Z^{p}(\mathcal{G}, V)$ and $B^{p}(\mathcal{G}, V)$ the $p$-cocycles and $p$-coboundaries of $\mathcal{G}$, respectively, with values in $V$.

Finally, $H^{p}(\mathcal{G}, V)=Z^{p}(\mathcal{G}, V) / B^{p}(\mathcal{G}, V)$ will denote the cohomology space of $\mathcal{G}$ of degree $p$ with values in $V$.

If $\mathcal{G}$ is a filtered Lie algebra, $\mathcal{G}=\bigcup_{i \in \mathbb{Z}} S_{i}$ and $V$ a filtered $\mathcal{G}$-module, $V=\bigcup_{i \in \mathbb{Z}} V_{i}$, we can consider the filtration $C^{j}(\mathcal{G}, V)=\bigcup_{k \in \mathbb{Z}} F_{k} C^{j}(\mathcal{G}, V)$ in the co-chains space where

$$
F_{k} C^{j}(\mathcal{G}, V)=\left\{c \in C^{j}(\mathcal{G}, V): c\left(x_{i_{1}}, \ldots, x_{i_{j}}\right) \in V_{i_{1}+\ldots+i_{j}+k}, x_{l} \in S_{l}\right\} .
$$

Then, as usual, we can provide the coboundary, cocycle and cohomology spaces, respectively, by the corresponding filtrations

$$
\begin{gathered}
Z^{j}(\mathcal{G}, V)=\bigcup_{k \in \mathbb{Z}} F_{k} Z^{j}(\mathcal{G}, V), \quad B^{j}(\mathcal{G}, V)=\bigcup_{k \in \mathbb{Z}} F_{k} B^{j}(\mathcal{G}, V), \\
H^{j}(\mathcal{G}, V)=\bigcup_{k \in \mathbb{Z}} F_{k} H^{j}(\mathcal{G}, V), \quad \text { with } F_{k} H^{j}(\mathcal{G}, V)=F_{k} Z^{j} / F_{k} B^{j} .
\end{gathered}
$$

By taking into consideration the gradation associated with any of these filtrations, we have

$$
\begin{aligned}
& C^{j}(\mathcal{G}, V)=\bigoplus_{i \in \mathbb{Z}} C_{i}^{j}(\mathcal{G}, V), \quad Z^{j}(\mathcal{G}, V)=\bigoplus_{i \in \mathbb{Z}} Z_{i}^{j}(\mathcal{G}, V), \\
& B^{j}(\mathcal{G}, V)=\bigoplus_{i \in \mathbb{Z}} B_{i}^{j}(\mathcal{G}, V), \quad H^{j}(\mathcal{G}, V)=\bigoplus_{i \in \mathbb{Z}} H_{i}^{j}(\mathcal{G}, V) .
\end{aligned}
$$




\section{Cohomology of Filiform Lie Algebras}

Let us recall that in a complex Lie algebra $\mathcal{G}$, one can consider the lower central series $(\mathcal{G})_{1}=\mathcal{G},(\mathcal{G})_{2}=[\mathcal{G}, \mathcal{G}], \ldots,(\mathcal{G})_{k}=\left[(\mathcal{G})_{k-1}, \mathcal{G}\right], \ldots$ It is said that $\mathcal{G}$ is filiform if $\operatorname{dim}(\mathcal{G})_{k}=n-k$ for $k \geqslant 2$, with $n=\operatorname{dim} \mathcal{G}$. Note that filiform Lie algebras are a subset of nilpotent Lie algebras.

If we denote by $L_{n}$ the model filiform Lie algebra of dimension $n$ and basis $\left\{e_{1}, \ldots, e_{n}\right\}$, then its bracket products are defined by $\left[e_{1}, e_{i}\right]=e_{i+1}$ for $2 \leqslant i \leqslant n-1$. For a general overview of this kind of algebras the reader can consult [1].

In this section we will describe cohomology spaces of the complex model Lie algebra $L_{n}$ having values in its adjoint module.

The algebra $L_{n}$ is filtered by considering the lower central series $L_{n}=\bigcup_{i \geqslant 1}\left(L_{n}\right)_{i}$, where $\left(L_{n}\right)_{1}=L_{n}$ and for $i \geqslant 2$ we have $\left(L_{n}\right)_{i}=\left\langle e_{i+1}, \ldots, e_{n}\right\rangle$. So, we can consider the following descending filtration in the 2-cocycles space of $L_{n}$ : $F_{k} Z^{2}\left(L_{n}, L_{n}\right)=\left\{\varphi \in Z^{2}\left(L_{n}, L_{n}\right): \varphi\left(\left(L_{n}\right)_{i},\left(L_{n}\right)_{j}\right) \subseteq\left(L_{n}\right)_{i+j+k}\right\}$. We have $Z^{2}\left(L_{n}, L_{n}\right)=\bigcup_{k \geqslant-1} F_{k} Z^{2}\left(L_{n}, L_{n}\right)$.

It is proved in [6] that the cohomology class of 2 -cocycles $\psi_{k, s}$ for $2 \leqslant k, 2 k \leqslant s \leqslant n$ defined by

$$
\begin{aligned}
& \psi_{k, s}\left(e_{i}, e_{i+1}\right)= \begin{cases}e_{s} & \text { if } i=k, \\
0 & \text { if } i \neq k,\end{cases} \\
& \psi_{k, s}\left(e_{1}, e_{i}\right)=0 \quad \text { for } 2 \leqslant i \leqslant n
\end{aligned}
$$

constitutes a basis of $F_{0} H^{2}\left(L_{n}, L_{n}\right)$. The definition of these cocycles will be written out in Section 4 after equation (4.1) in this paper.

\section{INTEGRABILITY IN THE COHOMOLOGY SPACE OF FILIFORM LIE ALGEBRAS}

Let us denote by $\mu$ the law of a Lie algebra $\mathcal{G}$. It is known that elements of $Z^{2}(\mathcal{G}, \mathcal{G})$ can be considered as infinitesimal deformations of $\mu$. Then we will say that $\psi \in$ $Z^{2}(\mathcal{G}, \mathcal{G})$ is linearity prolongable or integrable if the law defined by $[a, b]_{\psi}=\mu(a, b)+$ $\psi(a, b)$ for $a, b \in \mathcal{G}$ satisfies the Jacobi identity. The Lie algebra obtained starting from this law will be denoted by $\mathcal{G}_{\psi}$.

It is immediate that $\psi \in Z^{2}(\mathcal{G}, \mathcal{G})$ will be integrable if and only if

$$
(\psi \circ \psi)(a, b, c)=\psi(\psi(b, c), a)+\psi(\psi(c, a), b)+\psi(\psi(a, b), c)=0
$$

for all $a, b, c \in \mathcal{G}$. 
The study of the cohomology space of the model filiform Lie algebra will supply us a lot of information on filiform Lie algebras. According to Vergne (see [1]), every complex filiform Lie algebra is isomorphic to a Lie algebra $\left(L_{n}\right)_{\psi}$ for $\psi \in F_{0} H^{2}\left(L_{n}, L_{n}\right)$.

By considering the previous notation, the following result is valid, as we can see in $[7]$.

Proposition 3.1. The cocycle $\psi=\sum a_{k, s} \psi_{k, s} \in F_{0} H^{2}\left(L_{n}, L_{n}\right)$, where $a_{k, s} \neq 0$ for a pair $(k, s)$ such that $s=2 k<n$, is not integrable.

So, if $\psi \in F_{0} H^{2}\left(L_{n}, L_{n}\right)$ is integrable and defines a filiform Lie algebra, then either $\psi \in\left\langle\psi_{m, n}\right\rangle+F_{1} H^{2}\left(L_{n}, L_{n}\right)$ if $n$ is even, for $m=\frac{1}{2} n$, or $\psi \in F_{1} H^{2}\left(L_{n}, L_{n}\right)$ if $n$ is odd.

Proposition 3.2. The dimension of $F_{1} H^{2}\left(L_{n}, L_{n}\right)$ is $\frac{1}{4}\left(n^{2}-6 n+9\right)$ if $n$ is odd, or $\frac{1}{4}\left(n^{2}-6 n+8\right)$ if $n$ is even.

Proof. We know a basis of the space $F_{0} H^{2}\left(L_{n}, L_{n}\right)$ constituted by the cohomology classes of $\psi_{k, s}$, see (2.1). As $F_{1} H^{2}\left(L_{n}, L_{n}\right) \subset F_{0} H^{2}\left(L_{n}, L_{n}\right)$, it is sufficient to check that $F_{1} H^{2}\left(L_{n}, L_{n}\right)$ is generated by the cohomology classes of $\psi_{k, s}$ with $2 \leqslant k$, $2 k+1 \leqslant s \leqslant n$, because the linear independence of them is immediate, starting from the definition of $\psi_{k, s}$.

From now on, we will denote by $w$ the dimension of $F_{1} H^{2}\left(L_{n}, L_{n}\right)$. Let us consider the associated gradation: $F_{1} H^{2}\left(L_{n}, L_{n}\right)=\bigoplus_{i \geqslant 1} H_{i}^{2}\left(L_{n}, L_{n}\right)$. Then, if $\psi \in$ $F_{1} H^{2}\left(L_{n}, L_{n}\right)$, we can denote $\psi=\psi_{r}+\psi_{r+1}+\ldots+\psi_{p}$, where $\psi_{i} \in H_{i}^{2}\left(L_{n}, L_{n}\right)$, $\psi_{r} \neq 0, r \geqslant 1$.

The cocycle $\psi_{r}$ will be named the sill cocycle. It is easy to prove

Theorem 3.3. If $\psi$ is integrable, then its sill cocycle is also integrable.

The algebra $\left(L_{n}\right)_{\psi_{r}}$ is named the sill algebra of the Lie algebra $\left(L_{n}\right)_{\psi}$.

So, a first step to determine if $\psi \in F_{0} H^{2}\left(L_{n}, L_{n}\right)$ is integrable will be to obtain those $\psi_{i} \in H_{i}^{2}\left(L_{n}, L_{n}\right)$ for $i \geqslant 1$ which are integrable. 


\section{Structure of filiform Lie ALGebras}

In this section we will study the elements of $H_{i}^{2}\left(L_{n}, L_{n}\right)$ with $i \geqslant 0$, due to their important role in the structure of filiform Lie algebras.

By considering the natural gradation defined on $L_{n}$, if $\psi_{i} \in H_{i}^{2}\left(L_{n}, L_{n}\right)$ with $i \geqslant 0$, we can write

$$
\psi_{i}=\sum_{k \geqslant 2} a_{k, s} \psi_{k, s} \quad \text { with } s=2 k+i \leqslant n .
$$

For every pair $(k, s)$ with $2 \leqslant k, 2 k \leqslant s \leqslant n$, since $\psi_{k, s} \in Z^{2}\left(L_{n}, L_{n}\right)$, when the coboundary operator $d$ is applied, we obtain

$$
d \circ \psi_{k, s}\left(e_{1}, e_{i}, e_{j}\right)=0,
$$

that is

$$
\psi_{k, s}\left(e_{i}, e_{j}\right)=\left[e_{1}, \psi_{k, s}\left(e_{i}, e_{j-1}\right)\right]-\psi_{k, s}\left(e_{i+1}, e_{j-1}\right)
$$

for $2 \leqslant i<j \leqslant n$.

Starting from the definition of $\psi_{k, s}$ (2.1) and using (4.1), we have

$$
\begin{aligned}
& \text { If } k<i \text {, then } \psi_{k, s}\left(e_{i}, e_{j}\right)=0, \forall j>i . \\
& \text { If } k=i, \text { then } \psi_{k, s}\left(e_{i}, e_{j}\right)=\left(a d e_{1}\right)^{j-k-1}\left(e_{s}\right), \forall j>i . \\
& \text { If } k>i, \text { then } \psi_{k, s}\left(e_{i}, e_{j}\right)=\left(\begin{array}{c}
-1)^{k-i}\left(\begin{array}{c}
j-k-1 \\
k-i
\end{array}\right)\left(a d e_{1}\right)^{i+j-2 k-1} e_{s}, \\
\forall j>i, i+j-2 k-1 \geqslant 0 \text { and }
\end{array}\right. \\
& \qquad \psi_{k, s}\left(e_{i}, e_{j}\right)=0, \forall j>i, i+j-2 k-1<0 .
\end{aligned}
$$

So, we conclude that

$$
\psi_{k, s}\left(e_{i}, e_{j}\right)=(-1)^{k-i}\left(\begin{array}{c}
j-k-1 \\
k-i
\end{array}\right)\left(a d e_{1}\right)^{i+j-2 k-1} e_{s}
$$

for $2 \leqslant i \leqslant k<j \leqslant n, k-i \leqslant j-k-1$, and $\psi_{k, s}\left(e_{i}, e_{j}\right)=0$ otherwise, provided that $2 \leqslant i<j \leqslant n$.

By virtue of Proposition 3.1, for $n$ even and $i=0$, we can assume $\psi_{0}=a_{\frac{1}{2} n, n} \psi_{\frac{1}{2} n, n}$, because in the other case $\psi_{0}$ would not be integrable and thus it would not be of interest for our study. So,

$$
\begin{cases}\psi_{0}\left(e_{i}, e_{j}\right)=a_{\frac{1}{2} n, n}(-1)^{\frac{1}{2} n-i} e_{n}, & j=n+1-i, 2 \leqslant i \leqslant \frac{1}{2} n \\ \psi_{0}\left(e_{i}, e_{j}\right)=0 & \text { otherwise. }\end{cases}
$$


Fixing $i \geqslant 1, \psi_{i} \in H_{i}^{2}\left(L_{n}, L_{n}\right)$ for any $2 \leqslant q \leqslant\left[\frac{n-i}{2}\right]$ and denoting by $t_{k}$ the coefficients $a_{k, 2 k+i}$ we have

$$
\psi_{i}\left(e_{q}, e_{q+1}\right)=\sum_{s=2 k+i} t_{k} \psi_{k, s}\left(e_{q}, e_{q+1}\right)=t_{q} \psi_{q, 2 q+i}\left(e_{q}, e_{q+1}\right)=t_{q} e_{2 q+i} .
$$

According to (4.1), for $2 \leqslant q \leqslant\left[\frac{n-i}{2}\right]$ we also obtain

$$
\begin{aligned}
\psi_{i}\left(e_{q}, e_{q+2}\right) & =\sum_{l=q}^{\left[\frac{n-i}{2}\right]} t_{l} \psi_{l, 2 l+i}\left(e_{q}, e_{q+2}\right) \\
& =t_{q}\left[e_{1}, \psi_{q, 2 q+i}\left(e_{q}, e_{q+1}\right)\right]=t_{q} e_{2 q+1+i} .
\end{aligned}
$$

Finally, by using (4.2) for $2 \leqslant q \leqslant\left[\frac{n-i}{2}\right], q+2<p \leqslant n+1-q-i$, we have

$$
\psi_{i}\left(e_{q}, e_{p}\right)=\sum_{l=q}^{\left[\frac{n-i}{2}\right]} t_{l} \psi_{l, 2 l+i}\left(e_{q}, e_{p}\right)=\sum_{l=q}^{\left[\frac{p+q-1}{2}\right]}(-1)^{l-q} t_{l}\left(\begin{array}{c}
p-l-1 \\
l-q
\end{array}\right) e_{p+q+i-1} .
$$

Example 1. For $n=11$ and $i=1$, let us consider

$$
\psi_{1}=\sum_{k=2}^{5} a_{k, 2 k+1} \psi_{k, 2 k+1}=\sum_{k=2}^{5} t_{k} \psi_{k, 2 k+1} .
$$

Then $\mathcal{G}_{11}^{1}\left(t_{2}, t_{3}, t_{4}, t_{5}\right)=\left(L_{11}\right)_{\psi_{1}}$ has the following structure:

$$
\begin{array}{rlrl}
{\left[e_{1}, e_{k}\right]} & =e_{k+1} \text { for } 2 \leqslant k \leqslant 10, & & {\left[e_{2}, e_{9}\right]=\left(t_{2}-5 t_{3}+6 t_{4}-t_{5}\right) e_{11},} \\
{\left[e_{2}, e_{8}\right]=\left(t_{2}-4 t_{3}+3 t_{4}\right) e_{10},} & & {\left[e_{2}, e_{7}\right]=\left(t_{2}-3 t_{3}+t_{4}\right) e_{9},} \\
{\left[e_{2}, e_{6}\right]=\left(t_{2}-2 t_{3}\right) e_{8},} & {\left[e_{2}, e_{5}\right]=\left(t_{2}-t_{3}\right) e_{7},} \\
{\left[e_{2}, e_{4}\right]=t_{2} e_{6},} & {\left[e_{2}, e_{3}\right]=t_{2} e_{5},} \\
{\left[e_{3}, e_{8}\right]=\left(t_{3}-3 t_{4}+t_{5}\right) e_{11},} & {\left[e_{3}, e_{7}\right]=\left(t_{3}-2 t_{4}\right) e_{10},} \\
{\left[e_{3}, e_{6}\right]=\left(t_{3}-t_{4}\right) e_{9},} & {\left[e_{3}, e_{5}\right]=t_{3} e_{8},} \\
{\left[e_{3}, e_{4}\right]=t_{3} e_{7},} & {\left[e_{4}, e_{7}\right]=\left(t_{4}-t_{5}\right) e_{11},} \\
{\left[e_{4}, e_{6}\right]=t_{4} e_{10},} & {\left[e_{4}, e_{5}\right]=t_{4} e_{9},} \\
{\left[e_{5}, e_{6}\right]=t_{5} e_{11} .} &
\end{array}
$$




\section{VARIETY OF GRADED FILIFORM LIE ALGEBRAS}

As the space $F_{0} H^{2}\left(L_{n}, L_{n}\right)$ is generated by the cohomology class of 2-cocycles $\psi_{k, s}$ for $2 \leqslant k, 2 k \leqslant s \leqslant n$, the constants $a_{k, s}$ allow us to define an element $\psi \in$ $F_{0} H^{2}\left(L_{n}, L_{n}\right)$ as follows:

$$
\psi=\sum a_{k, s} \psi_{k, s} .
$$

Starting from Propositions 3.1 and 3.2 we can denote by $M^{n}$ the affine algebraic variety in the complex $(w+1)$-dimensional affine space $\mathbb{C}^{w+1}$, defined by the polynomials in $\mathbb{C}\left[a_{k, s}\right]$ obtained from considering $\psi \circ \psi\left(e_{q}, e_{p}, e_{r}\right)=0$ for all $2 \leqslant q<p<r \leqslant n$.

The decomposition $F_{0} H^{2}\left(L_{n}, L_{n}\right)=\bigoplus_{i \geqslant 0} H_{i}^{2}\left(L_{n}, L_{n}\right)$ allows us to conclude that $\bigcup_{i \geqslant 0} M_{i}^{n} \subseteq M^{n}$, where $M_{i}^{n}$ is the affine algebraic variety defined by the polynomials obtained by considering $\psi_{i} \circ \psi_{i}\left(e_{q}, e_{p}, e_{r}\right)=0$, for all $2 \leqslant q<p<r \leqslant n$ when $\psi_{i} \in H_{i}^{2}\left(L_{n}, L_{n}\right)$.

For $i=0$, Proposition 3.1 involves $M_{0}^{n}=\left\{\left(a_{\frac{1}{2} n, n}, 0, \ldots, 0\right)\right\}$. For $i \geqslant 1$, the space $H_{i}^{2}\left(L_{n}, L_{n}\right)$ is generated by the cohomology classes of $\psi_{k, s}$ with $s=2 k+i, k \geqslant 2$, $s \leqslant n$. So, to obtain $M_{i}^{n}$ it is sufficient to determine the constants $a_{k, 2 k+i}$ such that

$$
\psi_{i}=\sum_{k=2}^{\left[\frac{n-i}{2}\right]} a_{k, 2 k+i} \psi_{k, 2 k+i}=\sum_{k=2}^{\left[\frac{n-i}{2}\right]} t_{k} \psi_{k, 2 k+i}
$$

is integrable. That is, $\psi_{i} \circ \psi_{i}\left(e_{q}, e_{p}, e_{r}\right)=0$ for $2 \leqslant p<q<r \leqslant n$. But taking into consideration the structure of $\psi_{i} \in H_{i}^{2}\left(L_{n}, L_{n}\right)$ considered in the previous section, we have

$$
\psi_{i} \circ \psi_{i}\left(e_{q}, e_{p}, e_{r}\right)=P_{p, q, r}^{i, i} e_{p+q+r+2 i-2}
$$

where $P_{q, p, r}^{i, i}$ is a homogeneous polynomial of degree 2 in $\mathbb{C}\left[t_{2}, \ldots, t_{\left[\frac{n-i}{2}\right]}\right]$, provided $q+p+r+2 i-2 \leqslant n$, because it is identically zero in the other case.

So, the variety $M_{i}^{n}$ is defined by $\left\{P_{q, p, r}^{i, i}=0: q+p+r+2 i-2 \leqslant n\right\}$. Considering $a_{k, s}=0$ for $s \neq 2 k+i$, we have $M_{i}^{n} \subset M^{n}$.

Remark 1. If we denote by $Q_{q, p}^{i}$ with $i \geqslant 1$ the coefficient of the field $e_{q+p+i-1}$ in $\psi_{i}\left(e_{q}, e_{p}\right)$, that is, if

$$
\psi_{i}\left(e_{q}, e_{p}\right)=\sum_{l=q}^{\left[\frac{p+q-1}{2}\right]}(-1)^{l-q} t_{l}\left(\begin{array}{c}
p-l-1 \\
l-q
\end{array}\right) e_{p+q+i-1}=Q_{q, p}^{i} e_{p+q+i-1}
$$

then we have

$$
P_{q, p, r}^{i, i}=Q_{q, p}^{i} Q_{p+q+i-1, r}^{i}-Q_{q, r}^{i} Q_{q+r+i-1, p}^{i}+Q_{p, r}^{i} Q_{p+r+i-1, q}^{i}
$$


Example 2. By continuing with the notation from the previous example, the polynomials defining the variety $M_{1}^{11}$ are

$$
\begin{aligned}
& P_{3,4,6}^{1,1} \equiv 0, \\
& P_{3,4,5}^{1,1} \equiv 0, \\
& P_{2,3,6}^{1,1}=-3 t_{3}^{2}+5 t_{3} t_{4}+t_{3} t_{5}+2 t_{4} t_{2}-6 t_{4}^{2}+t_{4} t_{5}-2 t_{2} t_{5}, \\
& P_{2,4,5}^{1,1}=-4 t_{3} t_{4}+6 t_{4}^{2}-t_{4} t_{5}+2 t_{2} t_{5}-t_{3} t_{5}, \\
& P_{2,1,5}^{1,1}=t_{3} t_{4}+2 t_{2} t_{4}-3 t_{3}^{2}, \\
& P_{2,3,4}^{1,1}=t_{3} t_{4}+2 t_{2} t_{4}-3 t_{3}^{2} .
\end{aligned}
$$

Proposition 5.1. Under the conditions $i \geqslant 1,2 \leqslant q<p \leqslant n, p+q+i-1 \leqslant n-1$, we have

$$
Q_{q, p}^{i}=Q_{q+1, p}^{i}+Q_{q, p+1}^{i} .
$$

Pro of. It is immediate by using the basic properties of combinatorial numbers.

\section{Proposition 5.2.}

$$
P_{q, p, r}^{i, i}=P_{q, p, r-1}^{i, i}-P_{q, p+1, r-1}^{i, i}-P_{q+1, p, r-1}^{i, i}
$$

for $i \geqslant 1,2 \leqslant q<p<r, p+q+r+2 i-2 \leqslant n$.

Proof. It follows from the last result and from Remark 1. Indeed, if we denote $P_{q, p, r}^{i, i}-P_{q, p, r-1}^{i, i}+P_{q, p+1, r-1}^{i, i}+P_{q+1, p, r-1}^{i, i}=(a)+(b)+(c)$, where

$$
\begin{aligned}
(a) & =Q_{q, p}^{i} Q_{p+q-1+i, r}^{i}-Q_{q, p}^{i} Q_{p+q-1+i, r-1}^{i}+Q_{q, p+1}^{i} Q_{p+q+i, r-1}^{i}+Q_{q+1, p}^{i} Q_{p+q-i, r-1}^{i} \\
& =Q_{q, p}^{i}\left(Q_{p+q-1+i, r}^{i}-Q_{p+q-1+i, r-1}^{i}\right)+\left(Q_{q, p+1}^{i}+Q_{q+1, p}^{i}\right) Q_{p+q+i, r-1}^{i}
\end{aligned}
$$

due to $p+q+i \leqslant n-r+2-i \leqslant n-1$ and $p+q-2+2 i=n-r \leqslant n-1$, we can use the previous proposition and thus we have

$$
(a)=-Q_{q, p}^{i} Q_{p+q+i, r-1}^{i}+Q_{q, p}^{i} Q_{p+q+i, r-1}^{i}=0 .
$$

In a similar way it is easy to obtain

$$
\begin{aligned}
(b)= & -Q_{q, r}^{i} Q_{q+r+i-1, p}^{i}+Q_{q, r-1}^{i} Q_{q+r-2+i, p}^{i}-Q_{q, r-1}^{i} Q_{q+r-2+i, p+1}^{i} \\
& -Q_{q+1, r-1}^{i} Q_{q+r+i-1, p}^{i} \\
= & Q_{q, r-1}^{i}\left(Q_{q+r-2+i, p}^{n}-Q_{q+r-2+i, p+1}^{i}\right)+\left(-Q_{q, r}^{i}-Q_{q+1, r-1}^{i}\right) Q_{q+r+i-1, p}^{i} \\
= & \left(Q_{q, r-1}^{i}-Q_{q, r}^{i}-Q_{q+1, r-1}^{i}\right) Q_{q+r-1+i, p}^{i}=0
\end{aligned}
$$


and

$$
\begin{aligned}
(c)= & Q_{p, r}^{i} Q_{p+r+i-1, q}^{i}-Q_{p, r-1}^{i} Q_{p+r-2+i, q}^{i}+Q_{p+1, r-1}^{i} Q_{p+r-1+i, q}^{i} \\
& +Q_{p, r-1}^{i} Q_{p+r-2+i, q+1}^{i} \\
= & \left(Q_{p, r}^{i}+Q_{p+1, r-1}^{i}-Q_{p, r-1}^{i}\right) Q_{p+r-1+i, q}^{i}=0,
\end{aligned}
$$

which completes the proof.

Example 3. By continuing with Example 2, we have

$$
P_{3,4,6}^{1,1}=P_{2,3,5}^{1,1}-P_{2,4,5}^{1,1} ; \quad P_{3,4,5}^{1,1}=P_{2,3,4}^{1,1}
$$

Remark 2. By considering again the structure of cocycles in the spaces $H_{i}^{2}\left(L_{n}, L_{n}\right)$ for $i \geqslant 1$, it is easy to check that if $\bar{Q}_{q, p}^{i}$ denotes the coefficient of $e_{p+q+i-1}$ in $\bar{\psi}_{i}\left(e_{q}, e_{p}\right)$ for $\bar{\psi}_{i} \in H_{i}^{2}\left(L_{n-1}, L_{n-1}\right)$ we have $\bar{Q}_{q, p}^{i}=Q_{q, p}^{i}$ for all $2 \leqslant q<p$ with $p+q+i-1 \leqslant n-1$, where $Q_{q, p}^{i}$ represents the corresponding coefficient for $\psi_{i} \in H_{i}^{2}\left(L_{n}, L_{n}\right)$ such that $\psi_{i} \mid L_{n-1} \times L_{n-1}=\bar{\psi}_{i}$.

So, by denoting by $\bar{P}_{q, p, r}^{i, i}$ the associated polynomial with $\bar{\psi}_{i}$, we have verified that $\bar{P}_{q, p, r}^{i, i}=P_{q, p, r}^{i, i}$ for $2 \leqslant q<p<r \leqslant n$, with $p+q+r+2 i-2 \leqslant n-1$.

As a consequence of this note, if we define $\widehat{M}_{i}^{n-1}=M_{i}^{n-1}$ when $n-i$ is odd (that is $\left.\left[\frac{n-i}{2}\right]=\left[\frac{n-1-i}{2}\right]\right)$ and $\widehat{M}_{i}^{n-1}=M_{i}^{n-1} \times \mathbb{C}$ when $n-i$ is even, we have

$$
M_{i}^{n} \subseteq \widehat{M}_{i}^{n-1}
$$

So, an inductive method on the dimension $n$ can be used to obtain the variety $M_{i}^{n}$, once the variety $M_{i}^{n-1}$ is known. In fact, we have

Proposition 5.3. Given a fixed $i \geqslant 1$, we have $M_{i}^{n}=\widehat{M}_{i}^{n-1} \cap N_{i}^{n}$ for all $n \in \mathbb{N}$, where $N_{i}^{n}$ is the algebraic variety defined by

$$
\left\{P_{q, p, r}^{i, i}: p+q+r+2 i-2=n\right\} .
$$

Finally, we are going to see that the number of polynomials required to define $N_{i}^{n}$ can be notably reduced by using Proposition 5.2. As a consequence of it, any polynomial $P_{q, p, r}^{i, i}$ defining $N_{i}^{n}$ is a linear combination of polynomials $P_{q, p, p+1}^{i, i}$ with $q+2 p+1+2 i-2=n$, and of polynomials of $M_{i}^{n-1}$. To see it, we prove 
Theorem 5.4. The subvariety $M_{i}^{n} \subseteq \widehat{M}_{i}^{n-1}$ is defined by - $P_{2+2 k, \frac{n-2 i-1}{2}-k, \frac{n-2 i+1}{2}-k}^{i, i}$ for $0 \leqslant 3 k \leqslant \frac{n-2 i-7}{2}$, if $n$ is odd. - $P_{3+2 k, \frac{n-2 i-2}{2}-k, \frac{n-2 i}{2}-k}^{i, i}$ for $0 \leqslant 3 k \leqslant \frac{n-2 i-10}{2}$, if $n$ is even.

Proof. From Proposition 5.2 we have

$$
P_{q, p, r}^{i, i}=-P_{q, p+1, r-1}^{i, i}-P_{q+1, p, r-1}^{i, i}
$$

for $2 \leqslant q<p<r, p+q+r+2 i-2=n$, since $P_{q, p, r-1}^{i, i} \mid M_{i}^{n-1} \equiv 0$. Now, by recurrence on $q, p, r$ in (5.1), we obtain that any Jacobi polynomial defining $N_{i}^{n}$ in $\widehat{M}_{i}^{n-1}$ is a linear combination of $P_{q, p, p+1}^{i, i}$ with $q+2 p+1+2 i-2=n$.

\section{VARIETY OF FILIFORM LIE ALGEBRAS}

Let us consider $\psi=\sum a_{k, s} \psi_{k, s}=\psi_{l}+\ldots+\psi_{t} \in F_{0} H^{2}\left(L_{n}, L_{n}\right)$, where $\psi_{i}=$ $\sum_{k} a_{k, 2 k+i} \psi_{k, 2 k+i}$. We will study in this section the variety $M^{n}$, with $n$ arbitrary, defined starting from

$$
\psi \circ \psi=\psi_{l} \circ \psi_{l}+\sum_{i+j=2 l+1} \psi_{i} \circ \psi_{j}+\sum_{i+j=2 l+2} \psi_{i} \circ \psi_{j}+\ldots
$$

that is, $(\psi \circ \psi)\left(e_{q}, e_{p}, e_{r}\right)=0$ for $2 \leqslant q<p<r \leqslant n$.

If we denote by $P_{q, p, r}^{i, j}$ the polynomial obtained from $\left(\psi_{i} \circ \psi_{j}\right)\left(e_{q}, e_{p}, e_{r}\right)$, we have

$$
\begin{gathered}
(\psi \circ \psi)\left(e_{q}, e_{p}, e_{r}\right)=P_{q, p, r}^{l, l} e_{q+p+r+2 l-2}+\sum_{i+j=2 l+1} P_{q, p, r}^{i, j} e_{q+p+r+2 l+1-2} \\
+\ldots+\sum_{i+j=n+2-p-q-r} P_{q, p, r}^{i, j} e_{n}
\end{gathered}
$$

where $P_{q, p, r}^{i, j}=Q_{q, p}^{j} Q_{p+q+j-1, r}^{i}-Q_{q, r}^{j} Q_{r+q+j-1, p}^{i}+Q_{p, r}^{j} Q_{p+r+j-1, q}^{i}$ for any $i, j \geqslant 0$. In this expression, for $i \geqslant 1, Q_{q, p}^{i}$ follows the habitual notation from Remark 1 , while for $i=0$,

$$
Q_{q, p}^{0}= \begin{cases}(-1)^{\frac{1}{2} n-q} & \text { if } p=n+1-q \\ 0 & \text { otherwise }\end{cases}
$$

following the structure of $\psi_{0}$ from Section 4 . 
Proposition 6.1. $Q_{q, p}^{i}=Q_{q+1, p}^{i}+Q_{q, p+1}^{i}$ for $q+p+i-1 \leqslant n-1, i \geqslant 0$.

Pro of. For $i \leqslant 1$, the result follows from Proposition 5.1. When $i=0$, if $p+q<n$, then $Q_{q, p}^{0}=Q_{q+1, p}^{0}=Q_{q, p+1}^{0}=0$, whereas if $q+p=n$, then $Q_{q, p}^{0}=0=(-1)^{\frac{1}{2} n-q-1}+(-1)^{\frac{1}{2} n-q}=Q_{q+1, p}^{0}+Q_{q, p+1}^{0}$.

As a consequence, we have the following results. The proof of each of them is similar to the one made in Proposition 5.2.

Proposition 6.2. Given $i, j \geqslant 1$, if $q+p+r+i+j-2 \leqslant n$ with $q<p<r$, then

$$
P_{q, p, r}^{i, j}=P_{q, p, r-1}^{i, j}-P_{q, p+1, r-1}^{i, j}-P_{q+1, p, r-1}^{i, j} .
$$

Proposition 6.3. If $q+p+r+v-2=n$ with $q<p<r$, then

$$
P_{q, p, r}^{0, v}=-P_{q, p+1, r-1}^{0, v}-P_{q+1, p, r-1}^{0, v}
$$

Proposition 6.4. If $n$ is even and $a_{\frac{1}{2} n, n} \neq 0$, then $l=0$ with $\psi_{0}=a_{\frac{1}{2} n, n} \psi_{\frac{1}{2} n, n}$ in expression (6.1). In this case, holds $\psi_{v} \circ \psi_{0} \equiv 0 \forall v \geqslant 0$. Moreover, for $q<p<r$, if $r \neq n+2-q-p-v$, then

$$
\psi_{0} \circ \psi_{v}\left(e_{q}, e_{p}, e_{r}\right)=0 .
$$

P r o of. Under the hypothesis, by taking into consideration the structure of $\psi_{0}$, we have, for any $2 \leqslant q<p<r$, that $\psi_{v} \circ \psi_{0}\left(e_{q}, e_{p}, e_{r}\right)=\lambda \psi_{v}\left(e_{k}, e_{n}\right)$ for a certain $\lambda$ which is a multiple of $a_{\frac{1}{2} n, n}$ and a certain $k \geqslant 2$. As $v \geqslant 0$, we have $\psi_{v}\left(e_{k}, e_{n}\right)=0$, since $k+n-1>n$.

Moreover, if $r \neq n+2-q-p-v$, then

$$
\begin{aligned}
\psi_{0} \circ \psi_{v}\left(e_{q}, e_{p}, e_{r}\right)= & \psi_{0}\left(\psi_{v}\left(e_{q}, e_{p}\right), e_{r}\right)+\psi_{0}\left(\psi_{v}\left(e_{r}, e_{q}\right), e_{p}\right)+\psi_{0}\left(\psi_{v}\left(e_{p}, e_{r}\right), e_{q}\right) \\
= & Q_{q, p}^{v} \psi_{0}\left(e_{q+p+v-1}, e_{r}\right)+Q_{r, q}^{v} \psi_{0}\left(e_{r+q+v-1}, e_{p}\right) \\
& +Q_{p, r}^{v} \psi_{0}\left(e_{p+r+v-1}, e_{q}\right) \\
= & Q_{q, p}^{v} \cdot 0+Q_{r, q}^{v} \cdot 0+Q_{p, r}^{v} \cdot 0=0 .
\end{aligned}
$$

Remark 3. Denoting $L_{q, p, r}^{v}=\sum_{i+j=v} P_{q, p, r}^{i, j}$ for $v \geqslant 2, q<p<r$, we have the following results: 
- Either for $n$ odd or for $n$ even with $a_{\frac{1}{2} n, n}=0$, we have $l \geqslant 1$ in (6.1) and thus we can write

$$
(\psi \circ \psi)\left(e_{q}, e_{p}, e_{r}\right)=\sum_{v=2 l}^{n+2-q-p-r} L_{q, p, r}^{v} e_{p+q+r-2+v} .
$$

- If $n$ is even and $a_{\frac{1}{2} n, n} \neq 0$, then $l=0$ in (6.1) and Proposition 6.4, for $q<p<r$, implies

$$
(\psi \circ \psi)\left(e_{q}, e_{p}, e_{r}\right)=\sum_{v=2}^{n+2-q-p-r} L_{q, p, r}^{v} e_{p+q+r-2+v}+P_{q, p, r}^{0, n+2-q-p-r} e_{n} .
$$

So, under this notation, the following result follows as an immediate consequence of Proposition 6.2.

Theorem 6.5. For $2 \leqslant q<p<r \leqslant n, q+p+r+v-2 \leqslant n$, we have

$$
L_{q, p, r}^{v}=L_{q, p, r-1}^{v}-L_{q, p+1, r-1}^{v}-L_{q+1, p, r-1}^{v} .
$$

Example 4. Let us consider the algebra $\left(L_{11}\right)_{\psi}$, where

$$
\begin{aligned}
\psi= & \psi_{1}+\psi_{2}+\psi_{3}+\psi_{4}+\psi_{5}+\psi_{6}+\psi_{7} \\
= & \sum_{i=2}^{5} t_{i} \psi_{i, 2 i+1}+\sum_{i=2}^{4} h_{i} \psi_{i, 2 i+2}+\sum_{i=2}^{4} f_{i} \psi_{i, 2 i+3}+\sum_{i=2}^{3} g_{i} \psi_{i, 2 i+4} \\
& +\sum_{i=2}^{3} k_{i} \psi_{i, 2 i+5}+d_{2} \psi_{2,10}+b_{2} \psi_{2,11}
\end{aligned}
$$

defined by

$$
\begin{aligned}
{\left[e_{1}, e_{i}\right]=} & e_{i+1} \quad \text { for } 2 \leqslant i \leqslant 10 \\
{\left[e_{2}, e_{9}\right]=} & \left(t_{2}-5 t_{3}+6 t_{4}-t_{5}\right) e_{11}, \\
{\left[e_{2}, e_{8}\right]=} & \left(t_{2}-4 t_{3}+3 t_{4}\right) e_{10}+\left(h_{2}-4 h_{3}+3 h_{4}\right) e_{11}, \\
{\left[e_{2}, e_{7}\right]=} & \left(t_{2}-3 t_{3}+t_{4}\right) e_{9}+\left(h_{2}-3 h_{3}+h_{4}\right) e_{10} \\
& +\left(f_{2}-3 f_{3}+f_{4}\right) e_{11}, \\
{\left[e_{2}, e_{6}\right]=} & \left(t_{2}-2 t_{3}\right) e_{8}+\left(h_{2}-2 h_{3}\right) e_{9}+\left(f_{2}-2 f_{3}\right) e_{10} \\
& +\left(g_{2}-2 g_{3}\right) e_{11}, \\
{\left[e_{2}, e_{5}\right]=} & \left(t_{2}-t_{3}\right) e_{7}+\left(h_{2}-h_{3}\right) e_{8}+\left(f_{2}-f_{3}\right) e_{9}+\left(g_{2}-g_{3}\right) e_{10} \\
& +\left(k_{2}-k_{3}\right) e_{11},
\end{aligned}
$$




$$
\begin{aligned}
& {\left[e_{2}, e_{4}\right]=t_{2} e_{6}+h_{2} e_{7}+f_{2} e_{8}+g_{2} e_{9}+k_{2} e_{10}+d_{2} e_{11},} \\
& {\left[e_{2}, e_{3}\right]=t_{2} e_{5}+h_{2} e_{6}+f_{2} e_{7}+g_{2} e_{8}+k_{2} e_{9}+d_{2} e_{10}+b_{2} e_{11},} \\
& {\left[e_{3}, e_{8}\right]=\left(t_{3}-3 t_{4}+t_{5}\right) e_{11},} \\
& {\left[e_{3}, e_{7}\right]=\left(t_{3}-2 t_{4}\right) e_{10}+\left(h_{3}-2 h_{4}\right) e_{11},} \\
& {\left[e_{3}, e_{6}\right]=\left(t_{3}-t_{4}\right) e_{9}+\left(h_{3}-h_{4}\right) e_{10}+\left(f_{3}-f_{4}\right) e_{11},} \\
& {\left[e_{3}, e_{5}\right]=t_{3} e_{8}+h_{3} e_{9}+f_{3} e_{10}+g_{3} e_{11},} \\
& {\left[e_{3}, e_{4}\right]=t_{3} e_{7}+h_{3} e_{8}+f_{3} e_{9}+g_{3} e_{10}+k_{3} e_{11},} \\
& {\left[e_{4}, e_{7}\right]=\left(t_{4}-t_{5}\right) e_{11},} \\
& {\left[e_{4}, e_{6}\right]=t_{4} e_{10}+h_{4} e_{11},} \\
& {\left[e_{4}, e_{5}\right]=t_{4} e_{9}+h_{4} e_{10}+f_{4} e_{11},} \\
& {\left[e_{5}, e_{6}\right]=t_{5} e_{11} .}
\end{aligned}
$$

Computing $(\psi \circ \psi)\left(e_{2}, e_{3}, e_{4}\right)=(\psi \circ \psi)\left(e_{2}, e_{3}, e_{5}\right)=(\psi \circ \psi)\left(e_{2}, e_{4}, e_{5}\right)=(\psi \circ$ $\psi\left(e_{2}, e_{3}, e_{6}\right)=0$, and continuing with the usual notation, we conclude that the algebraic variety $M^{11} \subset \mathbb{C}^{16}$ is defined by the polynomials

$$
\begin{aligned}
L_{2,3,6}^{2}= & -3 t_{3}^{2}+5 t_{3} t_{4}+t_{3} t_{5}+2 t_{4} t_{2}-6 t_{4}^{2}+t_{4} t_{5}-2 t_{2} t_{5}, \\
L_{2,4,5}^{2}= & -4 t_{3} t_{4}+6 t_{4}^{2}-t_{4} t_{5}+2 t_{2} t_{5}-t_{3} t_{5}, \\
L_{2,3,5}^{2}= & t_{3} t_{4}+2 t_{2} t_{4}-3 t_{3}^{2}, \\
L_{2,3,5}^{3}= & 3 h_{2} t_{4}-7 t_{3} h_{3}+t_{3} h_{4}+3 h_{3} t_{4}+2 t_{2} h_{4}, \\
L_{2,3,4}^{2}= & t_{3} t_{4}+2 t_{2} t_{4}-3 t_{3}^{2}, \\
L_{2,3,4}^{3}= & 2 t_{2} h_{4}+3 h_{3} t_{4}-7 t_{3} h_{3}+t_{3} h_{4}+3 h_{2} t_{4}, \\
L_{2,3,4}^{4}= & 3 h_{3} h_{4}-4 h_{3}^{2}-8 t_{3} f_{3}+t_{3} f_{4}+6 f_{2} 3 t_{4}-f_{3} t_{5}+2 t_{2} f_{4}, \\
& \quad+3 h_{2} h_{4}+4 f_{2} t_{4}-2 f_{2} t_{5} .
\end{aligned}
$$

We observe that $L_{2,3,6}^{2}=L_{2,3,5}^{2}-L_{2,4,5}^{2}, L_{2,3,5}^{2}=L_{2,3,4}^{2}, L_{2,3,5}^{3}=L_{2,3,4}^{3}$. So, we can say that $M^{11}$ is also defined by $L_{2,4,5}^{2}, L_{2,3,4}^{2}, L_{2,3,4}^{3}$ and $L_{2,3,4}^{4}$.

Example 5. Let us consider the algebra $\left(L_{12}\right)_{\psi}$, where

$$
\begin{aligned}
\psi= & \psi_{0}+\psi_{1}+\psi_{2}+\psi_{3}+\psi_{4}+\psi_{5}+\psi_{6}+\psi_{7}+\psi_{8} \\
= & z \psi_{6,12}+\sum_{i=2}^{5} t_{i} \psi_{i, 2 i+1}+\sum_{i=2}^{5} h_{i} \psi_{i, 2 i+2}+\sum_{i=2}^{4} f_{i} \psi_{i, 2 i+3}+\sum_{i=2}^{4} g_{i} \psi_{i, 2 i+4} \\
& +\sum_{i=2}^{3} k_{i} \psi_{i, 2 i+5}+\sum_{i=2}^{3} d_{i} \psi_{i, 2 i+6}+b_{2} \psi_{2,11}+l_{2} \psi_{2,12},
\end{aligned}
$$


defined by

$$
\begin{aligned}
& {\left[e_{1}, e_{i}\right]=e_{i+1} \quad \text { for } 2 \leqslant i \leqslant 11,} \\
& {\left[e_{2}, e_{11}\right]=z e_{12} \text {, }} \\
& {\left[e_{2}, e_{10}\right]=\left(t_{2}-6 t_{3}+10 t_{4}-4 t_{5}\right) e_{12},} \\
& {\left[e_{2}, e_{9}\right]=\left(t_{2}-5 t_{3}+6 t_{4}-t_{5}\right) e_{11}+\left(h_{2}-5 h_{3}+6 h_{4}-h_{5}\right) e_{12},} \\
& {\left[e_{2}, e_{8}\right]=\left(t_{2}-4 t_{3}+3 t_{4}\right) e_{10}+\left(h_{2}-4 h_{3}+3 h_{4}\right) e_{11}} \\
& +\left(f_{2}-4 f_{3}+3 f_{4}\right) e_{12}, \\
& {\left[e_{2}, e_{7}\right]=\left(t_{2}-3 t_{3}+t_{4}\right) e_{9}+\left(h_{2}-3 h_{3}+h_{4}\right) e_{10}} \\
& +\left(f_{2}-3 f_{3}+f_{4}\right) e_{11}+\left(g_{2}-3 g_{3}+g_{4}\right) e_{12}, \\
& {\left[e_{2}, e_{6}\right]=\left(t_{2}-2 t_{3}\right) e_{8}+\left(h_{2}-2 h_{3}\right) e_{9}+\left(f_{2}-2 f_{3}\right) e_{10}} \\
& +\left(g_{2}-2 g_{3}\right) e_{11}+\left(k_{2}-2 k_{3}\right) e_{12}, \\
& {\left[e_{2}, e_{5}\right]=\left(t_{2}-t_{3}\right) e_{7}+\left(h_{2}-h_{3}\right) e_{8}+\left(f_{2}-f_{3}\right) e_{9}+\left(g_{2}-g_{3}\right) e_{10}} \\
& +\left(k_{2}-k_{3}\right) e_{11}+\left(d_{2}-d_{3}\right) e_{12} \text {, } \\
& {\left[e_{2}, e_{4}\right]=t_{2} e_{6}+h_{2} e_{7}+f_{2} e_{8}+g_{2} e_{9}+k_{2} e_{10}+d_{2} e_{11}+b_{2} e_{12},} \\
& {\left[e_{2}, e_{3}\right]=t_{2} e_{5}+h_{2} e_{6}+f_{2} e_{7}+g_{2} e_{8}+k_{2} e_{9}+d_{2} e_{10}+b_{2} e_{11}+l_{2} e_{12},} \\
& {\left[e_{3}, e_{10}\right]=-z e_{12} \text {, }} \\
& {\left[e_{3}, e_{9}\right]=\left(t_{3}-4 t_{4}+3 t_{5}\right) e_{12},} \\
& {\left[e_{3}, e_{8}\right]=\left(t_{3}-3 t_{4}+t_{5}\right) e_{11}+\left(h_{3}-3 h_{4}+h_{5}\right) e_{12} \text {, }} \\
& {\left[e_{3}, e_{7}\right]=\left(t_{3}-2 t_{4}\right) e_{10}+\left(h_{3}-2 h_{4}\right) e_{11}+\left(f_{3}-2 f_{4}\right) e_{12},} \\
& {\left[e_{3}, e_{6}\right]=\left(t_{3}-t_{4}\right) e_{9}+\left(h_{3}-h_{4}\right) e_{10}+\left(f_{3}-f_{4}\right) e_{11}+\left(g_{3}-g_{4}\right) e_{12} \text {, }} \\
& {\left[e_{3}, e_{5}\right]=t_{3} e_{8}+h_{3} e_{9}+f_{3} e_{10}+g_{3} e_{11}+k_{3} e_{12} \text {, }} \\
& {\left[e_{3}, e_{4}\right]=t_{3} e_{7}+h_{3} e_{8}+f_{3} e_{9}+g_{3} e_{10}+k_{3} e_{11}+d_{3} e_{12},} \\
& {\left[e_{4}, e_{9}\right]=z e_{12} \text {, }} \\
& {\left[e_{4}, e_{8}\right]=\left(t_{4}-2 t_{5}\right) e_{12} \text {, }} \\
& {\left[e_{4}, e_{7}\right]=\left(t_{4}-t_{5}\right) e_{11}+\left(h_{4}-t_{5}\right) e_{12},} \\
& {\left[e_{4}, e_{6}\right]=t_{4} e_{10}+h_{4} e_{11}+f_{4} e_{12} \text {, }} \\
& {\left[e_{4}, e_{7}\right]=t_{4} e_{9}+h_{4} e_{10}+f_{4} e_{11}+g_{4} e_{12},} \\
& {\left[e_{5}, e_{8}\right]=-z e_{12} \text {, }} \\
& {\left[e_{5}, e_{7}\right]=t_{5} e_{12} \text {, }} \\
& {\left[e_{5}, e_{6}\right]=t_{5} e_{11}+h_{5} e_{12},} \\
& {\left[e_{6}, e_{7}\right]=z e_{12} \text {. }}
\end{aligned}
$$


Computing now $(\psi \circ \psi)\left(e_{2}, e_{3}, e_{4}\right)=(\psi \circ \psi)\left(e_{2}, e_{3}, e_{5}\right)=(\psi \circ \psi)\left(e_{2}, e_{4}, e_{5}\right)=$ $(\psi \circ \psi)\left(e_{2}, e_{3}, e_{6}\right)=(\psi \circ \psi)\left(e_{3}, e_{4}, e_{5}\right)=(\psi \circ \psi)\left(e_{2}, e_{4}, e_{6}\right)=(\psi \circ \psi)\left(e_{2}, e_{5}, e_{6}\right)=$ $(\psi \circ \psi)\left(e_{2}, e_{3}, e_{7}\right)=(\psi \circ \psi)\left(e_{2}, e_{4}, e_{7}\right)=(\psi \circ \psi)\left(e_{2}, e_{3}, e_{8}\right)=0$, we obtain

$$
\begin{aligned}
& P_{2,3,8}^{0,1}=-2 t_{2} z-t_{5} z+3 t_{3} z, \\
& P_{2,4,7}^{0,1}=2 t_{2} z+t_{5} z-3 t_{3} z \\
& P_{2,3,7}^{0,2}=h_{4} z-2 h_{3} z \\
& L_{2,3,7}^{2}=-4 t_{2} t_{5}+9 t_{3} t_{4}+5 t_{3} t_{5}+2 t_{4} t_{2}+5 t_{4} t_{5}-3 t_{3}^{2}-16 t_{4}^{2}, \\
& P_{2,5,6}^{0,1}=-2 t_{2} z-t_{5} z+3 t_{3} z, \\
& P_{2,4,6}^{0,2}=-h_{4} z-2 h_{3} z \\
& L_{2,4,6}^{2}=-4 t_{3} t_{4}+10 t_{4}^{2}-4 t_{4} t_{5}+2 t_{2} t_{5}-4 t_{3} t_{5}, \\
& P_{2,3,6}^{0,3}=-2 f_{2} z+f_{4} z+f_{3} z, \\
& L_{2,3,6}^{2}=-3 t_{3}^{2}+5 t_{3} t_{4}+t_{3} t_{5}+2 t_{4} t_{2}-6 t_{4}^{2}+t_{4} t_{5}-2 t_{2} t_{5}, \\
& L_{2,3,6}^{3}=-7 t_{3} h_{3}+2 t_{2} h_{4}+3 t_{4} h_{2}+6 t_{3} h_{4}+t_{3} h_{5}+7 t_{4} h_{3}-16 t_{4} h_{4} \\
& +t_{4} h_{5}+2 h_{3} t_{5}+4 t_{5} h_{4}-2 t_{2} h_{5}-3 h_{2} t_{5} \\
& P_{3,4,5}^{0,2}=h_{4} z+2 h_{3} z \text {, } \\
& L_{3,4,5}^{2}=-4 t_{4}^{2}+3 t_{4} t_{5}+3 t_{2} t_{5} \text {, } \\
& P_{2,4,5}^{0,3}=2 f_{2} z-f_{4} z-f_{3} z, \\
& L_{2,4,5}^{2}=-4 t_{3} t_{4}+6 t_{4}^{2}-t_{4} t_{5}+2 t_{2} t_{5}-t_{3} t_{5}, \\
& L_{2,4,5}^{3}=-5 t_{3} h_{4}-t_{3} h_{5}-4 t_{3} h_{4}+16 t_{4} h_{4}-t_{4} h_{5}-2 t_{5} h_{3}-4 t_{5} h_{4}+2 t_{2} h_{5}+3 h_{2} t_{5} \text {, } \\
& L_{2,3,5}^{2}=t_{3} t_{4}+2 t_{2} t_{4}-3 t_{3}^{2} \text {, } \\
& L_{2,3,5}^{3}=3 h_{2} t_{4}-7 t_{3} h_{3}+t_{3} h_{4}+3 h_{3} t_{4}+2 t_{2} h_{4}, \\
& L_{2,3,5}^{4}=3 h_{3} h_{4}-4 h_{3}^{2}-8 t_{3} f_{3}+t_{3} f_{4}+6 f_{3} t_{4}-f_{3} t_{5}+2 t_{2} f_{4}+3 h_{2} h_{4}, \\
& +4 f_{2} t_{4}-2 f_{2} t_{5} \text {, } \\
& P_{2,3,4}^{0,5}=-2 k_{2} z-k_{3} z \\
& L_{2,3,4}^{2}=t_{3} t_{4}+2 t_{2} t_{4}-3 t_{3}^{2} \text {, } \\
& L_{2,3,4}^{3}=2 t_{2} h_{4}+3 h_{3} t_{4}-7 t_{3} h_{3}+t_{3} h_{4}+3 h_{2} t_{4} \text {, } \\
& L_{2,3,4}^{4}=3 h_{3} h_{4}-4 h_{3}^{2}-8 t_{3} f_{3}+t_{3} f_{4}+6 f_{3} t_{4}-f_{3} t_{5}+2 t_{2} f_{4}+3 h_{2} h_{4} \text {, } \\
& +4 f_{2} t_{4}-2 f_{2} t_{5} \text {, } \\
& L_{2,3,4}^{5}=-9 h_{3} f_{3}+3 h_{3} f_{4}-9 t_{3} g_{3}+t_{3} g_{4}+6 f_{3} h_{4}-f_{3} h_{5}+10 g_{3} t_{4}, \\
& -4 g_{3} t_{5}+2 t_{2} g_{4}+3 h_{2} f_{4}+4 f_{2} h_{4}-2 f_{2} h_{5}+5 g_{2} t_{4}-5 t_{5} g_{2} .
\end{aligned}
$$

The algebraic variety $M^{12} \subset \mathbb{C}^{21}$ is defined by the polynomials $L_{2,3,4}^{5}+P_{2,3,4}^{0,5}$, $L_{2,3,5}^{4}, L_{2,4,5}^{3}+P_{2,4,5}^{0,3}, L_{3,4,5}^{2}+P_{3,4,5}^{0,2}, L_{2,3,6}^{3}+P_{2,3,6}^{0,3}, L_{2,3,6}^{2}, L_{2,4,6}^{2}+P_{2,4,6}^{0,2}, L_{2,3,7}^{2}+P_{2,3,7}^{0,2}$, 
$P_{2,5,6}^{0,1}, P_{2,4,7}^{0,1}, P_{2,3,8}^{0,1}$, with polynomials defining $M^{11}$, that is $L_{2,3,4}^{4}, L_{2,3,4}^{3}, L_{2,3,4}^{2}$ and $L_{2,4,5}^{2}$.

In this case we obtain the following relations among them: $L_{2,3,5}^{4}=L_{2,3,4}^{4} ; P_{2,4,7}^{0,1}=$ $-P_{2,5,6}^{0,1} ; P_{2,3,8}^{0,1}=-P_{2,4,7}^{0,1} ; L_{2,3,6}^{3}+P_{2,3,6}^{0,3}=L_{2,3,5}^{3}-L_{2,4,5}^{3}-P_{2,4,5}^{0,3} ; L_{2,4,6}^{2}+P_{2,4,6}^{0,2}=$ $L_{2,4,5}^{2}-L_{3,4,5}^{2}-P_{3,4,5}^{0,2} ; L_{2,3,7}^{2}+P_{2,3,7}^{0,2}=L_{2,3,6}^{2}-L_{2,4,6}^{2}-P_{2,4,6}^{0,2}$.

So, we have deduced the following result, whose proof is a consequence of Re$\operatorname{mark} 2$.

Proposition 6.6. If $q+p+r+v-2 \leqslant n-1, v \geqslant 2$, then $L_{q, p, r}^{v}=\bar{L}_{q, p, r}^{v}$, where $\bar{L}_{q, p, r}^{v}$ is the polynomial obtained by considering the cocycle $\bar{\psi} \in F_{0} H^{2}\left(L_{n-1}, L_{n-1}\right)$ with $\psi \mid L_{n-1} \times L_{n-1}=\bar{\psi}$.

Note that this proposition is easily checkable in the previous two examples.

It is easy to see that the following result follows from Proposition 3.2: $\operatorname{dim} F_{1} H^{2}$ $\left(L_{n}, L_{n}\right)-\operatorname{dim} F_{1} H^{2}\left(L_{n-1}, L_{n-1}\right)$ is either $\frac{2 n-8}{4}$, if $n$ is even, or $\frac{2 n-5}{4}$, if $n$ is odd.

Let us now denote $\widehat{M}^{n-1}=M^{n-1} \times \mathbb{C}^{\frac{2 n-5}{4}}$, if $n$ is odd or $\widehat{M}^{n-1}=M^{n-1} \times \mathbb{C}^{\frac{2 n-8}{4}}$ if $n$ is even. The following result is then valid, as a consequence of the previous proposition.

Corollary 6.7. The following implications hold:

- If $n$ is odd, then $M^{n} \subseteq \widehat{M}^{n-1} \mid a_{\frac{n-1}{2}, n-1}=0$.

- If $n$ is even, then $M^{n} \mid a_{\frac{1}{2} n, n}=0 \subseteq \widehat{M}^{n-1}$.

If $N^{n}$ denotes the algebraic affine variety in $\mathbb{C}^{w+1}$ defined, respectively, by polynomials $L_{q, p, r}^{v}+P_{q, p, r}^{0, v}$ and $P_{l, m, t}^{0,1}$ if $n$ is even, or by $L_{q, p, r}^{v}$ if $n$ is odd, for $2 \leqslant q<p<r$, $q+p+r+v-2=n, v \geqslant 2,2 \leqslant l<m<t \leqslant n, l+m+t=n+1$, then the following theorem holds:

Theorem 6.8. $M^{n}=\widehat{M}^{n-1} \cap N^{n}$ for all $n \in \mathbb{N}$.

In the same way as we did in the previous section, the number of polynomials required to define $N^{n}$ can be also notably reduced. It can be made by using Theorem 6.5 if $n$ is odd, or the same theorem and Proposition 6.3 when $n$ is even. So, we have

Theorem 6.9. The subvariety $M^{n} \subseteq \widehat{M}^{n-1}$ is defined in the following way:

- if $n$ is odd, by polynomials $L_{n+1-v-2 p, p, p+1}^{v}$ for any $2 \leqslant v \leqslant n-7, \frac{n+1-v}{3}<$ $p \leqslant \frac{n-1-v}{2}$,

- if $n$ is even, by polynomials $L_{n+1-v-2 p, p, p+1}^{v}+P_{n+1-v-2 p, p, p+1}^{0, v}$ for $2 \leqslant v \leqslant n-7$, $\frac{n+1-v}{3}<p \leqslant \frac{n-1-v}{2}$ and $P_{n-2 l, l, l+1}^{0,1}$ with $\frac{1}{3} n<l \leqslant \frac{n-2}{2}$.

$\mathrm{Pr}$ o of. It is sufficient to use the recurrence method on $q, p, r$ from Theorem 6.5 and Proposition 6.3. 
Example 6. By applying this result to the previous examples, we can say that the variety $M^{12}$ is given by the polynomials defining $\widehat{M}^{11}$ (or $M^{11}$ ), that is $L_{2,3,4}^{4}, L_{2,3,4}^{3}$, $L_{2,3,4}^{2}, L_{2,4,5}^{2}$, and those defining $N^{12}: L_{2,3,4}^{5}+P_{2,3,4}^{0,5}, L_{2,4,5}^{3}+P_{2,4,5}^{0,3}, L_{3,4,5}^{2}+P_{3,4,5}^{0,2}$, $P_{2,5,6}^{0,1}$.

To compute all the above polynomials defining the variety $M^{n}$, any symbolic computation package can be used. Indeed, we have designed an algorithm which allows to obtain them. We will consider $n \geqslant 9$ due to the fact that for less values, no polynomial relation appears when describing the variety. A brief description of the algorithm follows.

\section{Algorithm.}

Input: The integer $n \geqslant 9$.

Output: The variety $M^{n}$ of laws of complex filiform Lie algebras of dimension $n$.

\section{Method:}

Step 1: Define polynomials $Q_{q, p}^{i}$, for $i \geqslant 1,2 \leqslant q<p \leqslant n, 3 \leqslant p+q+i-1 \leqslant n$. If $n$ is even, also define $Q_{q, p}^{0}$, for $2 \leqslant q \leqslant n, p=n+1-q$.

Step 2: For each $m$ with $9 \leqslant m \leqslant n$, use previous step to compute $P_{q, p, p+1}^{i, j}$, where $i, j \geqslant 1,2 \leqslant i+j \leqslant m-7, \frac{m+1-i-j}{3}<p \leqslant \frac{m-1-i-j}{2}, q=m+1-i-j-2 p$.

Step 3: For each $m$ with $9 \leqslant m \leqslant n$, compute $L_{q, p, p+1}^{v}=\sum_{i, j \geqslant 1, i+j=v} P_{q, p, p+1}^{i, j}$.

Step 4: If $n$ is even, for $\frac{n}{3}<l \leqslant \frac{n-2}{2}$ compute $P_{n-2 l, l, l+1}^{0,1}$ and for $2 \leqslant v \leqslant n-7$, $\frac{n+1-v}{3}<p \leqslant \frac{n-1-v}{2}, q=n+1-v-2 p$ compute $P_{q, p, p+1}^{0, v}$. If $n$ is odd, this stage is not needed.

Step 5: If $n$ is odd, the variety $M^{n}$ is defined by polynomials $L_{q, p, p+1}^{v}$. Otherwise, by polynomials $L_{n+1-v-2 p, p, p+1}^{v}+P_{n+1-v-2 p, p, p+1}^{0, v}, \stackrel{P}{n-2 l, l, l+1}^{0,1}$ and $L_{m+1-v-2 p, p, p+1}^{v}$ where $9 \leqslant m \leqslant n-1$.

To conclude the paper it is convenient to note that this reduction in the number of polynomials has been explicitly checked by us in the following cases: in dimension 12 we reduce from 17 polynomials to 8 ; in dimension 13 , from 25 to 11 ; in dimension 14 , from 48 to 18; in dimension 15, from 64 to 23; and so on, in dimension 18, from 203 to 55 . This proves that the algorithm significantly reduces the number of polynomials defining the variety. 


\section{References}

[1] M. Vergne: Cohomologie des algèbres de Lie nilpotentes. Application a l'étude de la variété des algèbres de Lie nilpotentes. Bull. Soc. Math. France 98 (1970), 81-116.

[2] N. Blackburn: On a special class of p-groups. Acta Math. 100 (1958), 45-92.

Zbl 0244.17011

[3] L. Boza Prieto, E. M. Fedriani Martel, and J. Núñez Valdés: A new method for classifying complex filiform Lie algebras. Applied Mathematics and Computation 121 (2001), 169-175.

Zbl 1021.17010

[4] M. Goze, Y.B. Khakimdjanov: Nilpotent and Solvable Lie Algebras. Handbook of Algebra, Vol. 2 (M. Hazewinkel, ed.). Elsevier, 2000. Zbl 0949.00006

[5] Y.B. Khakimdjanov: Varieties of Lie Algebras Laws. Handbook of Algebra, Vol. 2 (M. Hazewinkel, ed.). Elsevier, 2000.

Zbl 0949.00006

[6] M. Goze, Y.B. Khakimdjanov: Nilpotent Lie Algebras. Kluwer Academic Publishers, 1996.

Zbl 0845.17012

[7] Y. B. Khakimdjanov: Variété des lois d'algèbres de Lie nilpotentes. Geometriae Dedicata 40 (1991), 269-295.

Authors' address: Departamento de Geometría y Topología, Facultad de Matemáticas, Universidad de Sevilla, Aptdo. 1160, 41080-Sevilla, España, e-mails: cmgarcia@us.es, jnvaldes@us.es. 